\title{
Ambient bistable single dipole switching in a
}

\section{molecular monolayer}

Kang Cui, ${ }^{a, b}$ Kunal S. Mali, ${ }^{a}$ Dongqing Wu, ${ }^{c}$ Xinliang Feng, ${ }^{c}$ Klaus Müllen, ${ }^{c}$

Michael Walter, ${ }^{\mathrm{d}, e, f}$ Steven De Feyter, ${ }^{a}$ * Stijn F. L. Mertens ${ }^{\mathrm{a}, \mathrm{g}} *$

[a] Dr. K. Cui, Dr. S. Mali, Prof. S. De Feyter, Priv.-Doz. Dr. S. F. L. Mertens

KU Leuven, Department of Chemistry, Celestijnenlaan 200F, 3001 Leuven, Belgium

E-mail: steven.defeyter@kuleuven.be (S. De Feyter);

[b] Dr. K. Cui

University of Jinan, School of Chemistry and Chemical Engineering, Jinan 250022, P. R. China

[c] Dr. D. Q. Wu, Prof. X. L. Feng, Prof. K. Müllen,

Max Planck Institute for Polymer Research, 55128 Mainz, Germany

[d] Priv.-Doz. Dr. M. Walter

Freiburg Center for Interactive Materials and Bioinspired Technologies (FIT), Universität Freiburg, Georges-Köhler-Allee 105, 79110 Freiburg i. Br., Germany

[e] Priv.-Doz. Dr. M. Walter

Cluster of Excellence livMatS @ FIT

[f] Priv.-Doz. Dr. M. Walter

Fraunhofer IWM, Wöhlerstrasse 11, 79108 Freiburg i. Br., Germany

[g] Priv.-Doz. Dr. S. F. L. Mertens

Lancaster University, Department of Chemistry and Energy Lancaster, Bailrigg, LA1 4YB Lancaster, United Kingdom

E-mail: s.mertens@lancaster.ac.uk (S. F. L. Mertens)

This is the peer reviewed version of the article which has been published in final form at https://doi.org/10.1002/anie.202004016. This article may be used for non-commercial purposes in accordance with Wiley Terms and Conditions for Use of Self-Archived Versions. 


\begin{abstract}
We report a molecular dipole that self-assembles into highly ordered patterns at the liquid-solid interface and that can be switched at room temperature between a bright and a dark state at the single molecule level. Using a scanning tunnelling microscope (STM) under suitable bias conditions, we can write binary information at a density of up to $41 \mathrm{~Tb} / \mathrm{cm}^{2}\left(256 \mathrm{~Tb} / \mathrm{in}^{2}\right)$. The written information is stable during reading at room temperature, but can also be erased at will instantly by proper choice of tunnelling conditions. DFT calculations indicate that the contrast and switching mechanism originate from the stacking sequence of the molecular dipole, which is reoriented by the electric field between tip and substrate.
\end{abstract}

\title{
Main text
}

The appeal of molecular electronics lies in the easy bottom-up fabrication of nanostructures with functions that mimic, and sometimes surpass, those of traditional electronic components, while achieving ultimate spatial density. ${ }^{[1]}$ Over the past several decades, numerous molecular building blocks have been proposed ${ }^{[2]}$ to perform electronic functions that include quantised conduction, switching between conductance states, ${ }^{[3]}$ current amplification and rectification. ${ }^{[1]}$ For binary data storage at the molecular level, most results have been obtained in ultrahigh vacuum $(\mathrm{UHV}),{ }^{[4]}$ often combined with low temperatures (77K or less). ${ }^{[5]}$ In many cases, switching has been demonstrated in principle but was not achieved at the single molecule level, ${ }^{[5 \mathrm{c}, 5 \mathrm{~d}, 6]}$ or the switching units failed to form ordered layers. ${ }^{[4 \mathrm{~b}]}$ Among the desired properties of the ideal molecular electronics device feature ease of fabrication, low cost, sophisticated function, high stability and operation under ambient temperature and pressure. For a molecular memory, high information density, stability of the stored bits together with ease of writing, reading and erasing are of utmost importance. 
The system we propose is based on an organic salt that consists of a large discoid polyaromatic cation $\left(\mathrm{PQPC}_{14}{ }^{+}\right)$and a small inorganic anion (perchlorate, $\mathrm{ClO}_{4}^{-}$), Figure 1a. In recent work, we have demonstrated the rich supramolecular behaviour of this compound class, and were able to show transformations between 2- and 3-dimensional self-assembly and reversible electrocompression under electrochemical conditions, ${ }^{[7 a, b]}$ and the effect of anions on supramolecular structure formation. ${ }^{[7 \mathrm{c}]}$ The key difference between the molecular liquid-solid interface and the electrochemical environment is the presence of the electrochemical double layer, ${ }^{[8]}$ where typically a field on the order of up to $10^{9} \mathrm{~V} \mathrm{~m}^{-1}$ exists. This strong field leads to efficient ordering of permanently charged tectons, as Coulomb interactions dominate, and provides a decisive advantage over self-assembly at the organic solvent-solid interface. In the latter case, more subtle intermolecular interactions may lead to the formation of energetically nearly degenerate, and therefore polymorphic, structures. ${ }^{[9]}$ Here, we turn this seeming disadvantage to an advantage by performing STM in a low-dielectric solvent that confines the tectons with opposing charges to strongly bound ion pairs (in octanoic acid, with relative permittivity $\varepsilon=2.82$ at $25^{\circ} \mathrm{C},{ }^{[10]}$ we calculate a $4 \times 10^{25}$ stability constant for ion pair formation, see Supporting Information). This means that the ions are effectively "locked together" by the solvent and not free to move independently from each other, by contrast with the behaviour of solvated ions in a typical electrochemical environment. The electric field at the location of the tip being of the same order as in an electrochemical double layer then unlocks the possibility to switch these dipoles at the single molecular level and at room temperature, ${ }^{[11]}$ in a similar vein to electrostatic catalysis of covalent bond formation. ${ }^{[12]}$ 


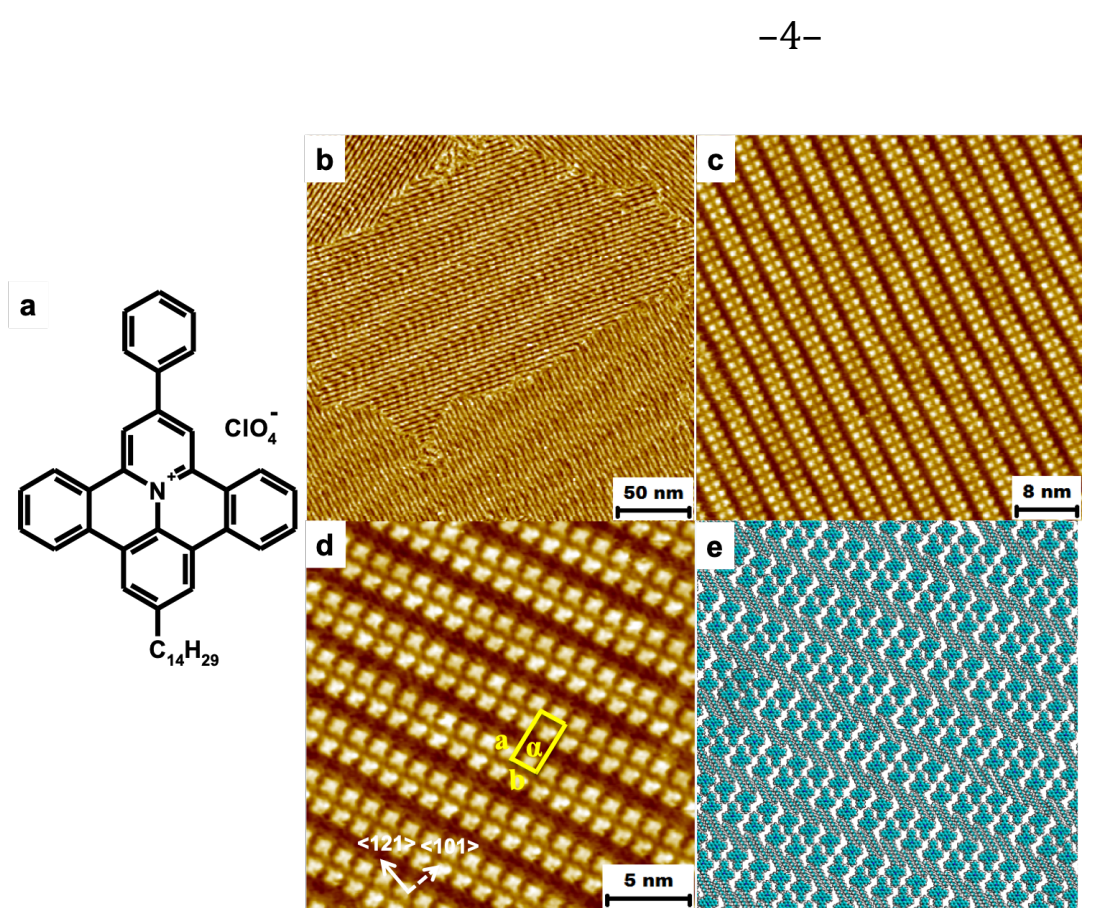

Figure 1. a) Molecular structure of $\mathrm{PQPC}_{14} \mathrm{ClO}_{4}$ (9-tetradecyl-2-phenylbenzo[8,9]quinolizino-[4,5,6,7-fed]phenanthridinylium perchlorate). b) Large scale, c) intermediate scale and d) high-resolution STM image of $\mathrm{Au}(111) / \mathrm{PQPC}_{14} \mathrm{ClO}_{4}(1 \mu \mathrm{M}$ in octanoic acid). Sample bias $U_{\mathrm{b}}=-0.8 \mathrm{~V}$, setpoint current $I_{\mathrm{t}}=80 \mathrm{pA}$. Unit cell parameters $a=(3.5 \pm 0.2) \mathrm{nm}, b=(1.4$ $\pm 0.2) \mathrm{nm}, \alpha=(87 \pm 3)^{\circ}$. e) Tentative model for molecular packing in panel $\mathrm{d}$.

Large- and intermediate-scale STM images, Figures $\mathbf{1 b}$ and $\mathbf{c}$, reveal that $\mathrm{PQPC}_{14} \mathrm{ClO}_{4}$ selfassembles into very large domains of several hundred square nanometres at room temperature at the octanoic acid/Au(111) interface; mild thermal annealing increases domain size further. Importantly for our purpose, virtually defect-free domains were obtained, indicating favourable kinetics of the self-assembly process and the absence of impurities with successfully competing adsorption characteristics. The herringbone reconstruction of the gold substrate is visible underneath the molecular adlayer, pointing at essentially incommensurate adsorption on the substrate, whose detailed features do not lower the symmetry of the adlayer. Within each domain, the tectons form straight rows, with exact molecular registry between the rows. In high-resolution images, Figure 1d, especially the latter feature makes it straightforward to identify every bright square as the fused polyaromatic core of the cation. The wider spacing of every double row accommodates the $n$-tetradecylchain that extends from the PQP core, leading to the model shown in Figure 1e. In the context of a molecular memory device, the tight 
packing, high level of simple rectangular order and absence of any spacer molecules are particularly attractive. ${ }^{[2]}$

Bias switching of the contrast is demonstrated in Figure 2. After imaging the area with initial tunnelling parameters $U_{\mathrm{b}}=-0.8 \mathrm{~V}, I_{\mathrm{t}}=70 \mathrm{pA}$ (as in Figure 1), a bias voltage pulse of $+0.5 \mathrm{~V}$ and $30 \mathrm{~ms}$ duration was applied at the indicated location. Following the pulse, imaging was continued with mildly negative bias $\left(U_{\mathrm{b}}=-0.25 \mathrm{~V}, I_{\mathrm{t}}=70 \mathrm{pA}\right)$. The effect of the positive bias pulse is visible as a highly localised attenuation of the contrast, which in Figure 2a is limited to two adjacent molecules. Closer inspection of the image still allows discerning their submolecular features, indicating that no vacancies have been created and no desorption has taken place. If the area with attenuated contrast is scanned using the initial tunnelling parameters $\left(U_{\mathrm{b}}=-0.8 \mathrm{~V}, I_{\mathrm{t}}=70 \mathrm{pA}\right)$, the original (all-bright) contrast is restored (vide infra).
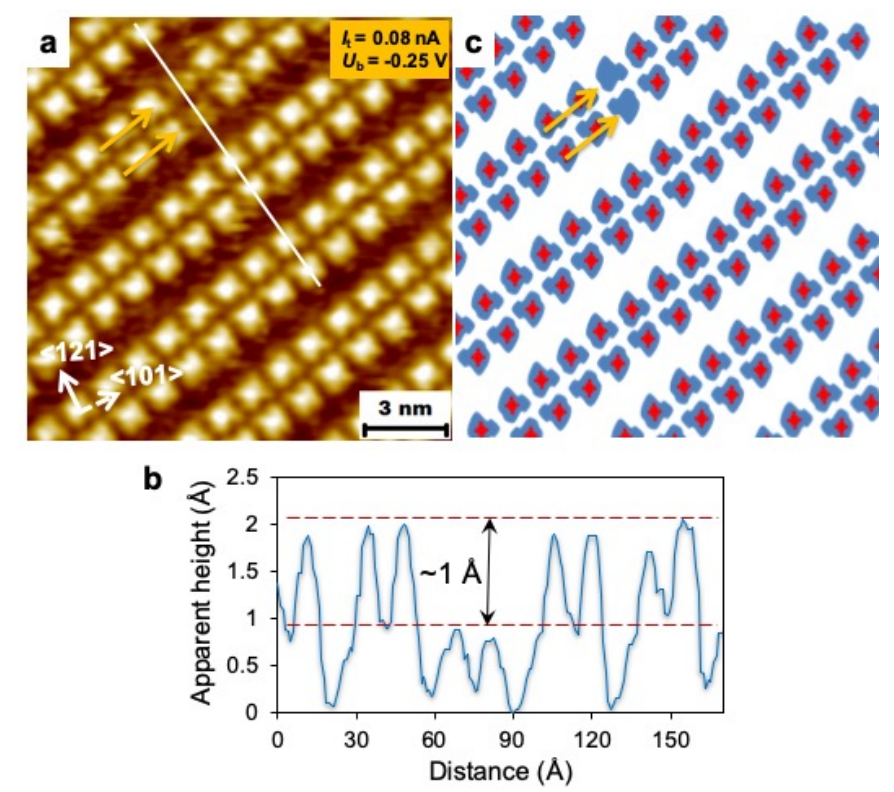

Figure 2. a) Bias switching of STM contrast by applying a $+0.5 \mathrm{~V}, 30 \mathrm{~ms}$ bias pulse at the indicated location. Imaging conditions $U_{\mathrm{b}}=-0.25 \mathrm{~V}, I_{\mathrm{t}}=80 \mathrm{pA}$. b) Apparent height profile along the white line in panel a. c) Two-tone schematic representation of contrast inversion. 
Next, we demonstrate that writing of the binary information is possible at the single molecule

level. Figure 3 shows a sequence of images where sequentially up to four molecules are switched (write) from bright to dark. The position of the switched molecules is simply determined by the position of the tip, provided that thermal drift is sufficiently low to achieve the required accuracy. As is obvious from the image sequence, no spurious switching ("bright" to "dark"), nor switching back occurs. After writing the 4 bits of information, the area shown was continuously imaged (read) for 30 min (corresponding to 11 full images), during which no changes in either direction were observed. Under erase conditions at $-0.8 \mathrm{~V}$ bias, all binary information units were reset to "bright" without exception.

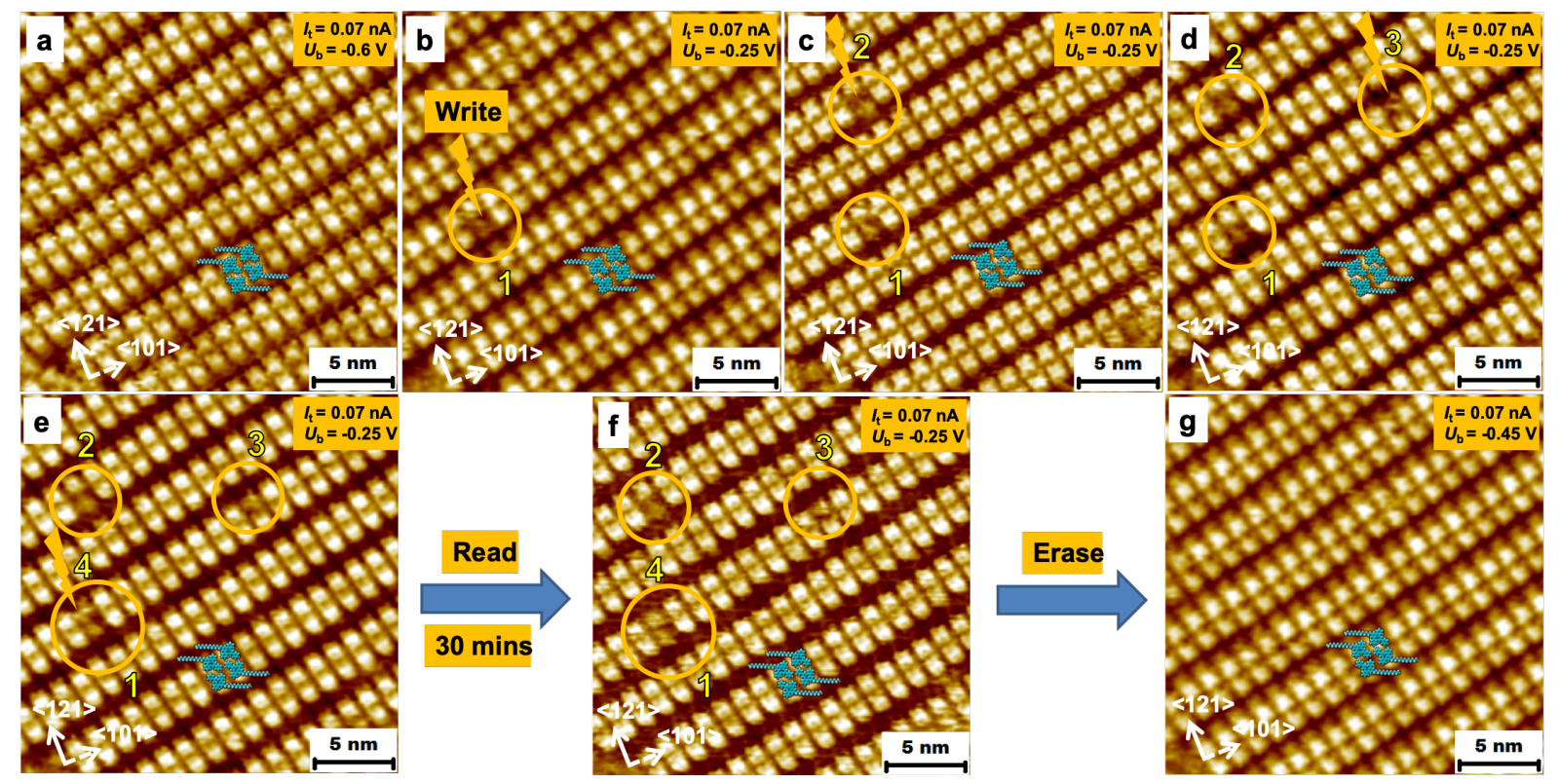

Figure 3. Single molecule writing, reading and erasing of binary information in a $\mathrm{PQPC}_{14} \mathrm{ClO}_{4}$ adlayer on $\mathrm{Au}(111)$. a) Adlayer before writing (all-zero); b-e) Sequential writing of up to four molecular bits of binary information; f) Stable reading of binary information; g) Complete erasing of written information with a single scan. Imaging conditions are indicated per panel. An impurity with irregular but stable contrast in the lower left corner of each image is used as spatial point of reference.

Bistability of the dipoles under reading conditions is an essential requirement for binary information storage, and is clearly demonstrated in Figure 3f. In order to establish the window 
of tunnelling conditions where stable reading is possible, continuous scanning of the all-bright adlayer was performed at low setpoint current ( $70 \mathrm{pA})$ while stepwise changing the sample bias until first signs of switching were observed (Figure S1 in Supporting Information). Similar experiments were carried out for switching back (Figure S2), leading to the curve shown in Figure 4. The expected hysteresis behaviour identifies a bistable region that is $150 \mathrm{mV}$ wide and whose steep sides lead to clear transitions between "bright" and "dark". The centre of the operational stability region is located at a substrate bias of $-0.25 \mathrm{~V}$; very low bias voltages (absolute values below $0.15 \mathrm{~V}$ ) cannot be used in practice as the resulting close proximity of the tip to the substrate disrupts the adlayer. The adlayer also possesses self-healing properties, which is demonstrated in Figure S3: if accidentally too large a bias voltage pulse is applied, leading to the switching of an unintentionally large number of molecules, prolonged scanning under "erasing" tunnelling conditions leads to an almost perfect recovery of the adlayer in the "all-zero" state, which propagates from the undisturbed sections of the rows.

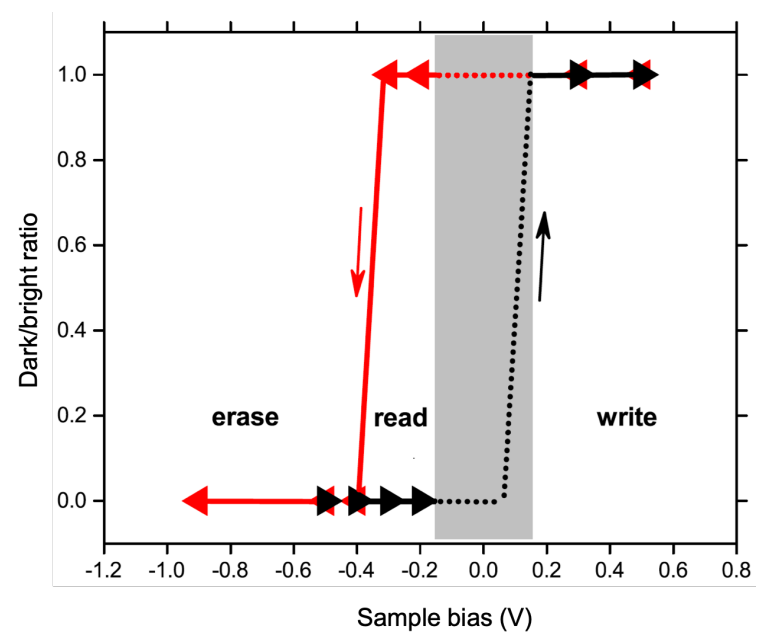

Figure 4. Ratio between "dark" and "bright" (" 1 " and " 0 ") as a function of sample bias. The clear hysteresis identifies bistability between -0.15 and $-0.3 \mathrm{~V}$, a precondition for stable reading. In the greyed out "blind region", $\pm 0.15 \mathrm{~V}$ around zero bias, imaging is not possible without disturbing the adlayer. 
In order to rationalise the experimentally observed switching behaviour, we performed density functional theory (DFT) calculations (see Methods), Figure 5. Under imaging conditions (tip positive), one of the most stable configurations of the ion pair is found for the $\mathrm{PQPC}_{14}{ }^{+}$cation adsorbed in a planar manner on the $\mathrm{Au}$ substrate, and with the $\mathrm{ClO}_{4}^{-}$anion stacked on top, Figure 5a, which represents the initial state ("bright") of the switching molecule (in the absence of the tip, calculations suggest that the $\mathrm{ClO}_{4}^{-}$relaxes to a position on the Au substrate, which is of no consequence for the switching we describe, Figures S4 and S5). The dipole moment of this vertically stacked ion pair amounts to 8.1 debye, more than twice the value determined for the Al-phthalocyanine with axial $\mathrm{Cl}$ ligand in other studies. ${ }^{[13]}$ The highest probability to switch individual dipoles was observed at a bias voltage pulse amplitude of 0.6 V (Figure S6). We hypothesise that during this pulse (tip negative), the perchlorate acquires sufficient energy to be inserted between the adjacent ordered alkyl chains, leading to a final state with a $\mathrm{ClO}_{4}^{-}$anion trapped between the $\mathrm{PQP}^{+}$tails as shown in Figure 5b. This configuration is one of several possibilities for trapping $\mathrm{ClO}_{4}^{-}$with very similar energies (Figure S7). Figure 5c shows the energy landscape that the $\mathrm{ClO}_{4}^{-}$anion experiences during its journey and Figure 5d the anion height along the trajectory between initial and final state. Importantly, there is a significant energy barrier for both the forward and the backward switching process, which explains the experimentally observed bistability (Figure S8). As the initial and final energies are state functions, a large number of trajectories with equivalent energy can be followed in a real experiment, all of which lead to the same final state ("dark"). Full inversion of the molecular dipole (flipping upside down) can be excluded, as desorption of the fused polyaromatic core of the $\mathrm{PQP}^{+}$cation from the $\mathrm{Au}(111)$ surface would require more than $2 \mathrm{eV}$ in energy, in clear disagreement with our experimental observations (Figure S9).

In line with this proposed mechanism, the change in contrast that is experimentally observed between the two states can be explained on the basis of the electronic density of states of our 
system, Figure 5e. During imaging, the positive tip (substrate bias negative) probes occupied states, i.e. states below the Fermi level. The figure indicates that occupied orbitals near the Fermi level are dominated by the high-energy state of the $\mathrm{ClO}_{4}^{-}$anion in the on-top configuration. Strikingly, contact with the gold surface lowers the energy of the $\mathrm{ClO}_{4}{ }^{-}$frontier electrons in the side position (Figure S4) and more so for trapping of $\mathrm{ClO}_{4}{ }^{-}$between the alkyl chains. The energy landscape shown in Figure $\mathbf{5 b}$ is very shallow as long as the perchlorate is on top of the fused ring system of the $\mathrm{PQPC}_{14}{ }^{+}$cation, leading to high mobility of the anion. ${ }^{[7 c]}$ During normal imaging (not switching), the perchlorate will follow the positive tip within the periphery of the $\mathrm{PQP}^{+}$fused ring system, enhancing the brightness of the latter. Once a perchlorate is trapped by the $\mathrm{C}_{14}$-chains after switching, it becomes electronically invisible and is hindered to move on top of the $\mathrm{PQP}^{+}$ring system, yielding the "dark" state. This picture is confirmed by the simulated STM pictures in Figure $\mathbf{5}$ f where large values of the apparent height are found only if perchlorate is in the on-top position.
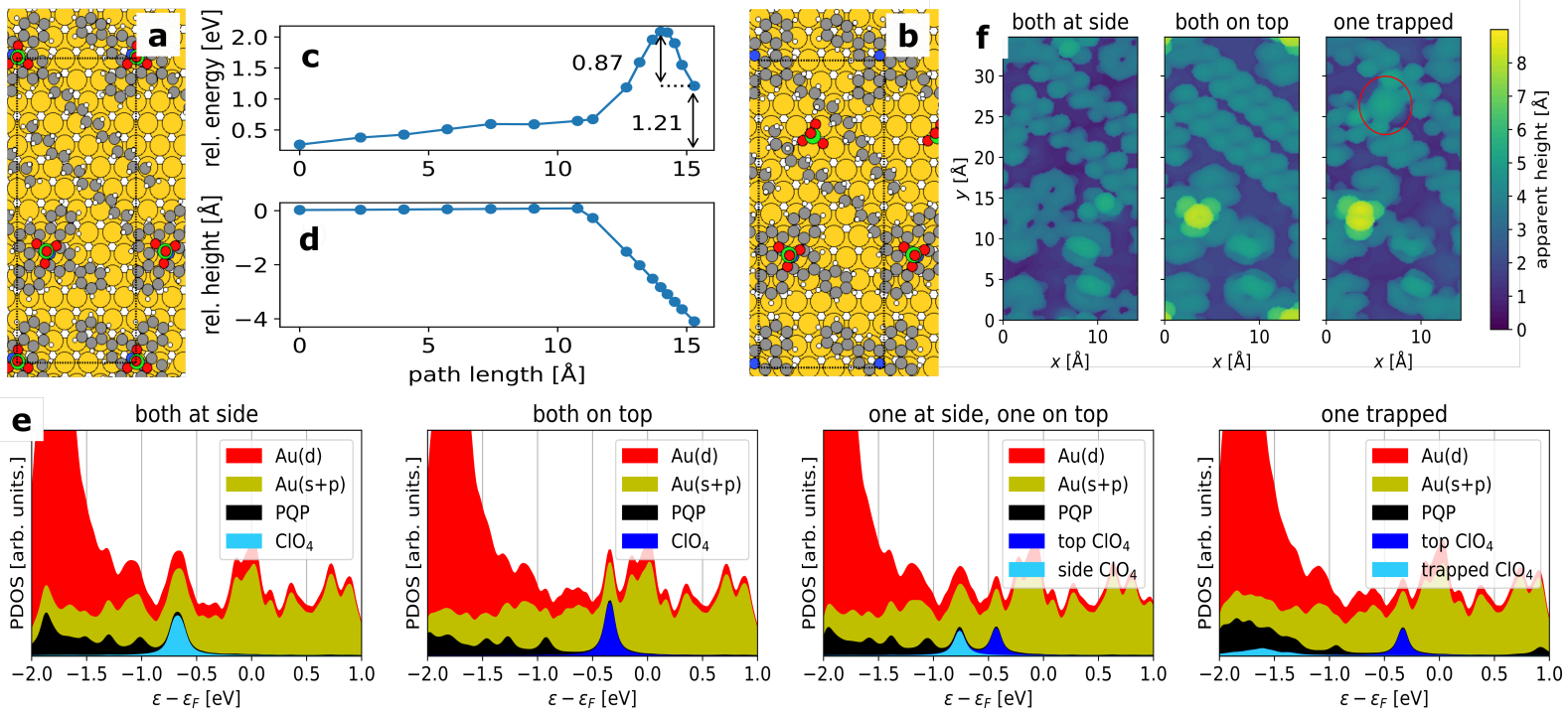

Figure 5. a) Initial and b) final configuration, and c,d) reaction path of the $\mathrm{ClO}_{4}{ }^{-}$anion to move from an on-top position to a trapped position between the $\mathrm{C}_{14} \mathrm{H}_{29}$ tails. The corresponding relative energies and barriers are shown. e) Projected density of states (PDOS) relative to the Fermi level $\varepsilon_{F}$ for different positions of the $\mathrm{ClO}_{4}{ }^{-}$anions (see Figure $\mathrm{S} 4$ for the corresponding configurations). f) Simulated STM images within the unit cell; the position of the trapped $\mathrm{ClO}_{4}{ }^{-}$ anion is indicated with a circle. 
As the very high information density is achieved in a single-component system, without the use of any passive spacer molecule, there is a limit to the number of adjacent molecules that can be switched with retention of the adlayer structure. Consequently, the achievable information density is somewhat lower than the calculated $41 \mathrm{Tbit} / \mathrm{cm}^{2}$. The fact that adjacent molecules can be switched without disturbing the rest of the adlayer (as shown in Figure 3E) may enable practically achievable binary information densities between 20 and $30 \mathrm{Tbit} / \mathrm{cm}^{2}$.

The liquid medium in which the self-assembly of the dipoles and their switching takes place is at first sight remote from existing technologies that almost exclusively operate in the solid state. However, we believe that the low-dielectric organic liquid involved, or analogous compounds, are unproblematic regarding technological implementation. Octanoic acid, a naturally occurring fatty acid, has very low volatility (normal boiling point $239^{\circ} \mathrm{C}$ ), ${ }^{[10]}$ is non-toxic and not corrosive towards materials that are commonly used in the electronics industry. In a closed system, the amounts necessary to provide the liquid interface film would be very limited: based on the footprint of the $\mathrm{PQP}^{+}$cation, less than $10 \mu \mathrm{L}$ of a $10^{-5} \mathrm{M}$ solution is sufficient to produce an ordered monolayer on a $1 \mathrm{~cm}^{2}$ gold substrate - corresponding to a liquid film thickness of $<100 \mu \mathrm{m}$. Such small amounts of non-conducting liquid would be perfectly compatible with technological alternatives to STM, such as cross-wire arrays. ${ }^{[14]}$

In summary, we have demonstrated a molecular binary information storage concept that is operable at ambient temperatures and pressures down to the single molecule level, at a theoretical information density of $41 \mathrm{Tbit} / \mathrm{cm}^{2}$. This achievement brings practicable molecular electronics a step closer. 


\section{Acknowledgements}

This work was supported by the Fund for Scientific Research-Flanders (FWO-Vlaanderen) under EOS 30489208, KU Leuven internal funds and by the Austrian Science Fund (FWF, project I3256-N36). We thank the NEMO cluster at the University of Freiburg for computational resources.

\section{Experimental}

$\mathrm{PQPC}_{14} \mathrm{ClO}_{4}$ was synthesised following published procedures. ${ }^{[15]} 20 \mu \mathrm{L}$ of a $10^{-6} \mathrm{M}$ solution in octanoic acid (99\%, Sigma-Aldrich) was placed on freshly flame-annealed Au/mica and introduced in an Agilent 5100 STM or a Bruker STM combination. Imaging was performed at room temperature $\left(20 \pm 2{ }^{\circ} \mathrm{C}\right)$ in air using mechanically cut $80 / 20 \mathrm{Pt} / \mathrm{Ir}$ tips made from $0.25 \mathrm{~mm}$ wire (Advent Research Materials Ltd., UK).

Density functional theory calculations were performed within the projector augmented wave formalism ${ }^{[16]}$ as implemented in GPAW. ${ }^{[17]}$ The smooth wave functions were represented on real space grids using a grid spacing of $h=0.2 \AA$. The exchange-correlation energy was described in the generalized gradient approximation as proposed by Perdew, Burke and Ernzerhof ${ }^{[18]}$ and additional van der Waals interactions were considered. ${ }^{[19]}$ The $\mathrm{Au}(111)$ surface was modelled by two layers of gold (140 Au atoms) fixed at the experimental lattice constant of the fcc structure. Periodic boundary conditions where applied in directions of the surface plane and the Brillioun zone was described by the $\Gamma$ point for the energetics and sampled by a $(3,3,1)$ k-point grid to obtain PDOS and simulate STM images. ${ }^{[20]}$ The molecules were allowed to relax without symmetry constraints and were considered to be relaxed when all forces fell below $0.05 \mathrm{eV} / \AA$. 


\section{References}

[1] A. Aviram, M. A. Ratner, Chem. Phys. Lett. 1974, 29, 277-283.

[2] J. L. Zhang, J. Q. Zhong, J. D. Lin,W. P. Hu, K. Wu, G. Q. Xu, A. T. S. Wee, W. Chen, Chem. Soc. Rev. 2015, 44, 2998-3022.

[3] a) N. J. Tao, Phys. Rev. Lett. 1996, 76, 4066-4069; b) T. Albrecht, S. F. L. Mertens, J. Ulstrup, J. Am. Chem. Soc. 2007, 129, 9162-9167; c) Y. He, E. Borguet, Angew. Chem. Int. Ed. 2007, 46, 6098-6101; Angew. Chem. 2007, 119, 6210-6213.

[4] a) L. C. Liu, S. Y. Liu, X. Chen, C. Li, J. Ling, X. Q. Liu, Y. X. Cai, L. Wang, Sci. Rep. 2013, 3, 3062; b) J. Liu, C. Li, X. Q. Liu, Y. Lu, F. F. Xiang, X. L. Qiao, Y. X. Cai, Z. P. Wang, S. Q. Liu, L. Wang, ACS Nano 2014, 8, 12734-12740.

[5] a) Y. L. Huang, Y. H. Lu, T. C. Niu, H. Huang, S. Kera, N. Ueno, A. T. S. Wee, W. Chen, Small 2012, 8, 1423-1428; b) J. L. Zhang, J. L. Xu, T. C. Niu, Y. H. Lu, L. Liu, W. Chen, J. Phys. Chem. C 2014, 118, 1712-1718; c) H. Yanagi, K. Ikuta, H. Mukai, T. Shibutani, Nano Lett. 2002, 2, 951-955; d) T. G. Gopakumar, F. Muller, M. Hietschold, J. Phys. Chem. C 2006, $110,6051-6059$.

[6] S. B. Lei, K. Deng, Y. L. Yang, Q. D. Zeng, C. Wang, J. Z. Jiang, Nano Lett. 2008, 8, 18361843.

[7] a) K. Cui, O. Ivasenko, K. S. Mali, D. Q. Wu, X. L. Feng, K. Müllen, S. De Feyter, S. F. L. Mertens, Chem. Commun. 2014, 50, 10376-10378; b) K. Cui, K. S. Mali, O. Ivasenko, D. Q Wu, X. L Feng, M. Walter, K. Müllen, S. De Feyter, S. F. L. Mertens, Angew. Chem. Int. Ed. 2014, 53, 12951-12954; Angew. Chem. 2014, 126, 13165-13168; c) K. Cui, K. S. Mali, O. Ivasenko, D. Q. Wu, X. L. Feng, M. Walter, K. Müllen, S. De Feyter, S. F. L. Mertens, Small 2017, 13, 1702379.

[8] C. H. Hamann, A. Hamnett, W. Vielstich, Electrochemistry, 2nd Edition, Wiley-VCH, Weinheim, Germany, 2007. 
[9] K. S. Mali, D. Q Wu, X. L Feng, K. Müllen, M. V. Auweraer, S. De Feyter, J. Am. Chem. Soc. 2011, 133, 5686-5688.

[10] CRC Handbook of Chemistry and Physics (Ed. D. R. Lide), 85th Edition, CRC Press, Boca Raton, FL, USA, 2004.

[11] a) S. F. L. Mertens, Adsorption and Self-Organization of Organic Molecules under Electrochemical Control, in Encyclopedia of Interfacial Chemistry: Surface Science and Electrochemistry (Ed. K. Wandelt), Elsevier, Amsterdam, The Netherlands, 2018, Vol. 4, 1323; b) K. Cui, I. Dorner, S. F. L. Mertens, Curr. Opin. Electrochem. 2018, 8, 156-163.

[12] a) A. C. Aragonès, N. L. Haworth, N. Darwish, S. Ciampi, N. J. Bloomfield, G. G. Wallace, I. Diez-Perez, M. L. Coote, Nature 2016, 531, 88-91; b) S. Shaik, D. Mandal, R. Ramanan, Nat. Chem. 2016, 8, 1091-1098.

[13] H. Fukagawa, S. Hosoumi, H. Yamane, S. Kera, N. Ueno, Phys. Rev. B 2011, 83, 085304. [14] J. G. Kushmerick, J. Naciri, J. C. Yang, R. Shashidhar, Nano Lett. 2003, 3, 897-900.

[15] a) X. L. Feng, V. Marcon, W. Pisula, M. R. Hansen, J. Kirkpatrick, F. Grozema, D. Andrienko, K. Kremer, K. Müllen, Nat. Mater. 2009, 8, 421-426; b) D. Q. Wu, W. Pisula, V. Enkelmann, X. L. Feng, K. Muellen, J. Am. Chem. Soc. 2009, 131, 9620-9621; c) D. Q. Wu, L. J. Zhi, G. Bodwell, G. L. Cui, N. Tsao, K, Müllen, Angew. Chem. Int. Ed. 2007, 46, 5417-5420; Angew. Chem. 2007, 119, 5513-5516.

[16] P. E. Blochl, Phys. Rev. B 1994, 50, 17953-17979.

[17] a) J. J. Mortensen, L. B. Hansen, K. W. Jacobsen, Phys. Rev. B 2005, 71, 035109; b) J. Enkovaara1, C. Rostgaard, J. J. Mortensen, J. Chen, M. Dułak, L. Ferrighi, J. Gavnholt, C. Glinsvad, V. Haikola, H. A. Hansen, H. H. Kristoffersen et al., J. Phys.-Condens. Mat. 2010, $22,253202$.

[18] J. P. Perdew, K. Burke, M. Ernzerhof, Phys. Rev. Lett. 1996, 77, 3865-3868.

[19] A. Tkatchenko, M. Scheffler, Phys. Rev. Lett. 2009, 102, 073005. 
[20] J. Tersoff, D. R. Hamann, Phys. Rev. Lett. 1983, 50, 1998-2001.

\section{Table of Contents}

Molecular flip-flop: By pulsing the tip voltage in a scanning tunnelling microscope, individual molecules in a monolayer of a polyaromatic salt can be switched reversibly from a bright (" 0 ") to a dark (" 1 ") state, at room temperature and outside of vacuum. The information density of this single molecule binary memory can reach up to 41 terabit per $\mathrm{cm}^{2}$.

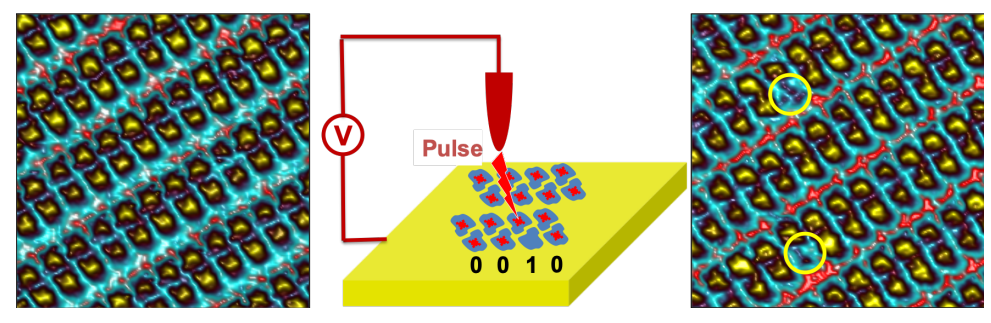

Keywords: DFT calculations, molecular electronics, scanning tunneling microscopy, selfassembled monolayers, single molecule switches 